\title{
Heat-transfer-based detection of L-nicotine, histamine, and serotonin using molecularly imprinted polymers as biomimetic receptors
}

\author{
M. Peeters • P. Csipai • B. Geerets • A. Weustenraed • \\ B. van Grinsven • R. Thoelen - J. Gruber • \\ W. De Ceuninck • T. J. Cleij • F. J. Troost • P. Wagner
}

Received: 4 March 2013 /Revised: 11 April 2013 /Accepted: 25 April 2013 /Published online: 18 May 2013

(C) Springer-Verlag Berlin Heidelberg 2013

\begin{abstract}
In this work, we will present a novel approach for the detection of small molecules with molecularly imprinted polymer (MIP)-type receptors. This heat-transfer method (HTM) is based on the change in heat-transfer resistance imposed upon binding of target molecules to the MIP nanocavities. Simultaneously with that technique, the impedance is measured to validate the results. For proof-ofprinciple purposes, aluminum electrodes are functionalized with MIP particles, and L-nicotine measurements are
\end{abstract}

This paper is dedicated to Professor Franz Dickert on the occasion of his 70 th birthday.

\footnotetext{
M. Peeters $(\bowtie) \cdot$ P. Csipai $\cdot$ B. Geerets $\cdot$ A. Weustenraed $\cdot$ B. van Grinsven · R. Thoelen • W. De Ceuninck · T. J. Cleij • P. Wagner

Institute for Materials Research, Hasselt University,

Wetenschapspark 1,

3590 Diepenbeek, Belgium

e-mail: marloes.peeters@uhasselt.be

\section{P. Csipai · J. Gruber}

Instituto de Química, Universidade de São Paulo,

Av. Prof. Lineu Prestes, 748,

CEP 05508-000 São Paulo, SP, Brazil
}

\section{R. Thoelen}

Xios University College Limburg, Agoralaan-Building H,

3590 Diepenbeek, Belgium

W. De Ceuninck $\cdot$ P. Wagner

Division IMOMEC, IMEC vZw, Wetenschapspark 1,

3590 Diepenbeek, Belgium

\section{F. J. Troost}

Division of Gastroenterology-Hepatology,

Department of Internal Medicine,

Maastricht University Medical Center, Minderbroedersberg 4-6,

6211 LK Maastricht, The Netherlands performed in phosphate-buffered saline solutions. To determine if this could be extended to other templates, histamine and serotonin samples in buffer solutions are also studied. The developed sensor platform is proven to be specific for a variety of target molecules, which is in agreement with impedance spectroscopy reference tests. In addition, detection limits in the nanomolar range could be achieved, which is well within the physiologically relevant concentration regime. These limits are comparable to impedance spectroscopy, which is considered one of the state-of-the-art techniques for the analysis of small molecules with MIPs. As a first demonstration of the applicability in biological samples, measurements are performed on saliva samples spiked with L-nicotine. In summary, the combination of MIPs with HTM as a novel readout technique enables fast and low-cost measurements in buffer solutions with the possibility of extending to biological samples.

Keywords Molecularly imprinted polymers (MIPs) · Heat-transfer resistance $\left(R_{\mathrm{th}}\right) \cdot$ Impedance spectroscopy . L-Nicotine $\cdot$ Heat-transfer method (HTM)

\section{Introduction}

Molecularly imprinted polymers (MIPs) are synthetic receptors which can bind their target as specifically and selectively as an enzyme [1, 2]. The focus of the imprinting strategy was originally on small organic molecules, but the technique has been extended to biological molecules such as proteins [3] and living cells [4-6]. As compared to natural antibodies, the advantages of MIPs are the low-cost and straightforward production [7], unlimited shelf life [8], and 
good thermal and chemical stability [9]. Depending on the required morphology, the MIP synthesis can be performed with various methods including bulk polymerization [10], emulsion polymerization [11], and electropolymerization [12]. We will focus here on the most commonly used method, which is bulk polymerization since it is straightforward and widely applicable.

In recent years, the interest from the bio-analytical field has increased rapidly because MIPs are extremely suitable for the detection of chemical targets in complex matrices such as urine, blood, and saliva [13]. For separation purposes, MIPs can be readily used by packing them directly into separation columns [14]. The main drawbacks of chromatographic techniques are the often time-consuming measurements and the requirement of expensive equipment $[15$, 16]. The incorporation of MIPs into sensing devices therefore remains challenging. In literature, the majority of the sensor platforms is based on gravimetric detection [17] and electronic readout platforms $[18,19]$. In contrast, electrochemical techniques are inexpensive, but the analysis is often complicated. There are a few examples of MIP measurements in biological samples such as in human blood plasma [20], intestinal fluid [21], blood serum [22, 23], and urine [23]. These measurements are all in vitro; until now, little has been reported about their application in living organisms. Hoshino et al. [24] studied the behavior of molecularly imprinted polymer nanoparticles for mellitin in vivo. With fluorescent labeling of the target, they could determine that the nanoparticles could effectively capture the mellitin from the bloodstream of mice. Furthermore, the particles were demonstrated to be nontoxic to cultured cells (fibrosarcoma cells) over a concentration range of $3-3,000 \mu \mathrm{g} / \mathrm{ml}$. This is a very promising result for future in vivo measurements, but the fluorescent technique is costly and not label free.

In this article, we will focus on detection by means of differential heat transfer resistance. For the heat transfer method (HTM), only two thermocouples, a proportional-integralderivative (PID) controller and an adjustable heat source, are required, ensuring a straightforward sensor platform and lowcost detection. This approach has been recently applied for the screening of single nucleotide polymorphisms in DNA fragments [25], which makes it a valuable tool in mutation analysis. To our knowledge, HTM has not been employed yet for small-molecule detection with MIP receptors. There are some examples of thermometric MIP sensors in literature, but they are based on the reaction heat developed upon binding and not on the heat transfer properties of the MIP layer [26-28]. As a proof-of-principle experiment, we studied the detection of Lnicotine (Fig. 1) with the HTM concept.

L-Nicotine is the major addictive substance in tobacco [29]. Consumption of tobacco has proven to result in a higher risk for the development of cancer [30, 31] and disorders, e.g., pulmonary disease [32] and atherosclerosis [33]. Thoelen et a<smiles>CN1CCCC1c1cccnc1</smiles><smiles>CN1C(=O)CCC1c1cccnc1</smiles>

b<smiles>NCCC1=CCNC=N1</smiles><smiles>NC(CC1=CCNC=N1)C(=O)O</smiles>

C

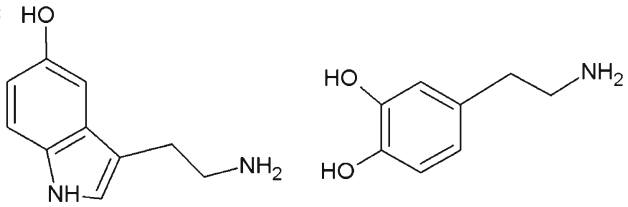

Fig. 1 Chemical structures of a L-nicotine, its metabolite cotinine b histamine, its precursor histidine c serotonin, and its competitor dopamine

al. [34] integrated MIP particles for L-nicotine into an impedimetric sensor setup and measured a series of concentrations in phosphate-buffered saline (PBS) solutions. This MIP, based on the monomer methacrylic acid (MAA), will be used for further measurements described within this article.

The detection of histamine and serotonin in biological fluids has been reported previously with MIP receptors in combination with impedimetric readout $[20,21]$. With some slight modifications to the setup (Fig. 2), we can simultaneously measure the impedance signals and heat transfer resistance for direct validation of the results. First, proof-ofprinciple measurements are conducted with L-nicotine in PBS. Subsequently, similar experiments are performed with histamine and serotonin to show the principle for a variety of targets. For the applicability of the sensor platform in biological samples, spiked L-nicotine samples in saliva were studied, and a dose-response curve was constructed. In summary, we will demonstrate the fast and low-cost detection of small molecules in buffer solutions with MIP

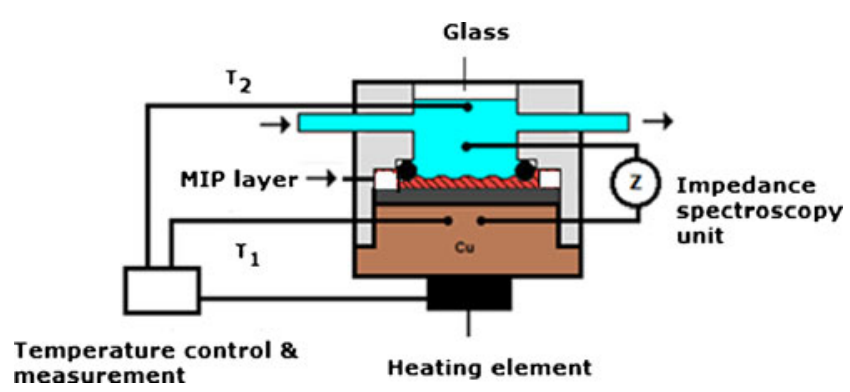

Fig. 2 Schematic illustration of the general concept of the measuring setup. The temperature of the copper block, $T_{1}$, is strictly controlled at $37.00 \pm 0.02{ }^{\circ} \mathrm{C}$. The heat flows from the copper block through the MIP layer to the liquid, where $T_{2}$ is measured. Simultaneously with the temperature, the impedance is monitored 
receptors in combination with HTM. Since this method has the possibility of extending to biological samples, it offers a huge potential for analytical research.

\section{Experimental}

\section{Materials}

Ethylene glycol dimethacrylate (EGDM), MAA, acrylic acid (AA), acrylamide, and dimethylsulfoxide were obtained from Acros (Geel, Belgium). Prior to polymerization, the stabilizers in EGDM, MAA, and AA were removed by filtration over alumina. The initiator azobisisobutyronitrile (AIBN) was purchased from Fluka (Buchs, Switzerland). As templates, Lnicotine, histamine, and serotonin (Figs. 1a-c) were used. LNicotine was obtained from Acros, while serotonin and histamine were purchased from Alfa Aesar (Karlsruhe, Germany). All solvents were of analytical grade and used without further purification.

\section{MIP synthesis}

The MIP for L-nicotine was synthesized as follows: First, a mixture of $12.5 \mathrm{mmol}$ MAA, $72 \mathrm{mmol}$ EGDM, and $0.61 \mathrm{mmol}$ AIBN was dissolved in $7 \mathrm{ml}$ hexane together with $6.41 \mathrm{mmol}$ of the template molecule L-nicotine. The solution was degassed with $\mathrm{N}_{2}$ and polymerized in a thermostatic water bath at $60^{\circ} \mathrm{C}$ for $72 \mathrm{~h}$. After polymerization, the polymer was ground, and the L-nicotine was removed by Soxhlet extraction with methanol $(48 \mathrm{~h})$, a mixture of acetic acid/acetonitrile (1/1) (48 h), and again, methanol (12 h). A non-imprinted polymer (NIP) was synthesized according to the same procedure, but without the presence of the target molecule. The synthesis procedure for the MIPs for serotonin and histamine is described in detail in refs [20] and [10].

Electrode preparation for the thermal resistance and impedance measurements

For the heat transfer resistance and impedance measurements, $1 \times 1-\mathrm{cm}^{2}$ aluminum substrates were spincoated with conductive $\mathrm{OC}_{1} \mathrm{C}_{10}$-polyphenylenevinylene (PPV). This PPV derivative, serving as an adhesive layer, was synthesized via the sulfinyl precursor route [35]. Subsequently, MIP and NIP particles were applied to the surface with a polydimethylsiloxane stamp. By heating above the glass transition temperature of $120{ }^{\circ} \mathrm{C}$, the powder is allowed to sink partially into the adhesive layer. After cooling, the surface is rinsed with isopropanol to remove excessive powder and ensure that the particles are strongly fixated into the layer [34]. To demonstrate an equal load of the
MIP and NIP electrode, the sensor surface was studied with an Axiovert 40 inverted optical microscope (Carl Zeiss). With optical microscopy in combination with image processing (software by Image $\mathrm{J}$ of the National Institute of Health, Bethesda, USA), the MIP (25 $\pm 2 \%)$ and NIP (24 \pm $3 \%$ ) were found to have nearly identical particle loadings which is necessary to perform differential measurements.

\section{Design of sensor setup}

The general concept for the measuring setup is shown in Fig. 2. The aluminum substrates, functionalized with MIP and NIP particles, were horizontally mounted into a Perspex flowcell with an internal volume of $110 \mu \mathrm{l}$. Silver paste ensured good thermal contact between the copper and the substrate. Two miniature thermocouples (type K; diameter, $500 \mu \mathrm{m}$, TC Direct, the Netherlands) monitored the temperature of the copper backside contact $\left(T_{1}\right)$ and the temperature of the fluid $\left(T_{2}\right) 1.7 \mathrm{~mm}$ above the chip surface. The temperature $T_{1}$ was strictly controlled at $37.00 \pm 0.02{ }^{\circ} \mathrm{C}$ with a homemade PID controller (parameters: $P=10, I=5, D=0.1)$. Hereby, the temperature inside the human body is mimicked. For the generated heat flow, a power resistor (22 $\Omega$, MPH20, Farnell, Belgium) was used which was mechanically attached to the copper block with heat-conductive paste. Simultaneously with the temperature, the impedance response was measured in a frequency range of $100 \mathrm{~Hz}$ to $100 \mathrm{kHz}$ with ten frequencies per decade and a scanning speed of $5.7 \mathrm{~s}$ per sweep. The amplitude of the $\mathrm{AC}$ voltage was fixed to $10 \mathrm{mV}$ under open circuit conditions. All measurements were performed under static conditions [25]

Sample preparation in PBS solutions for L-nicotine, histamine, and serotonin

For a proof-of-principle experiment, the detection of L-nicotine was performed in PBS buffer ( $\mathrm{pH}=7.4)$. PBS buffer was used to simulate the ionic strength of biological samples. The Lnicotine concentrations were varied from $100 \mathrm{nM}$ to $1.0 \mathrm{mM}$ to ensure a wide concentration regime is analyzed. To test the selectivity, the same concentrations were prepared with cotinine in PBS. This procedure was repeated for histamine and serotonin. In the case of histamine, histidine served as an analog, while for serotonin, its competitor dopamine was selected.

Sample preparation of spiked saliva samples

As a next step, saliva samples were analyzed. To collect the saliva, a nonsmoker test person deposited saliva in a sterilized Falcon tube. The saliva was centrifuged immediately for $10 \mathrm{~min}$ with a speed of $10,000 \mathrm{rpm}$ and the 
supernatant subsequently filtered with a $1-\mu \mathrm{m}$ syringe filter. The obtained saliva samples were split into several aliquots. One aliquot was kept unaltered, thereby serving as a control fluid. The other aliquots were spiked with L-nicotine concentrations of $0.25,0.5,1.0,2.5$, and $10.0 \mathrm{mM}$.

\section{Results and discussion}

Proof of principle: L-nicotine measurements in buffer

The MIP- and NIP-functionalized aluminum chips were mounted into the flow cell, which was subsequently filled with PBS of $\mathrm{pH}$ 7.4. The flow cell was placed in an environment with a stable ambient temperature of $19 \pm 0.02{ }^{\circ} \mathrm{C}$. The temperature of the copper, $T_{1}$, was strictly controlled at $37 \pm$ $0.02{ }^{\circ} \mathrm{C}$ by the PID controller. When $T_{2}$ reached a stable level, increasing concentrations of L-nicotine in PBS $(0.05-10 \mu \mathrm{M})$ were added. Between each addition, the sensor was left to stabilize for at least $15 \mathrm{~min}$. The time dependence of $T_{2}$ for a measurement with the MIP functionalized electrode is shown in Fig. 3.

In PBS, $T_{2}$ stabilizes at $35.2 \pm 0.02{ }^{\circ} \mathrm{C}$. After adding increasing concentrations of L-nicotine, a drop in the temperature $T_{2}$ is observed. However, to analyze the layer properties before and after binding of the target exactly, we should study not only the effect on temperature $T_{1}$ and $T_{2}$ but also the power $(P)$. Therefore, we propose to investigate the thermal resistance $\left(R_{\mathrm{th}}\right)$, as was done previously for the detection of point mutations in DNA [25]. This is defined as follows:

$R_{\mathrm{th}}=\frac{T_{1}-T_{2}}{P}$

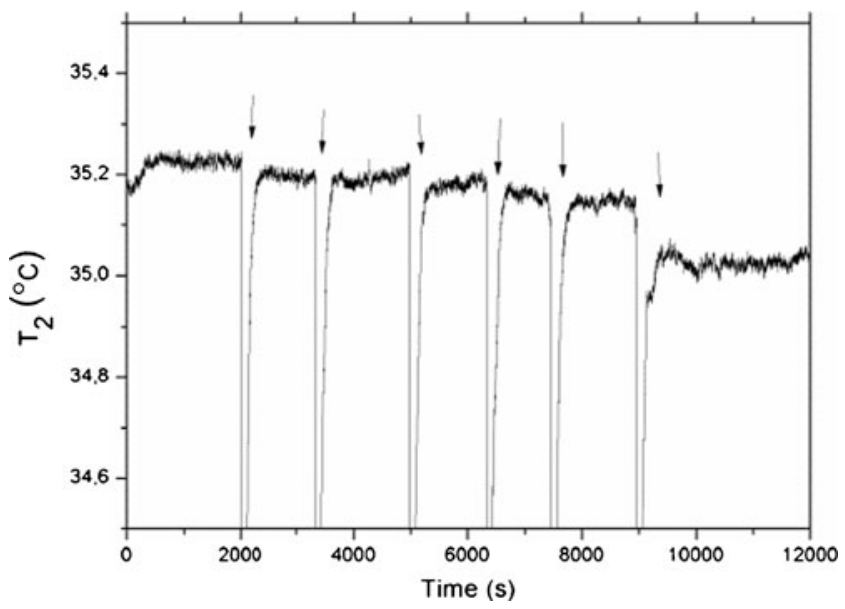

Fig. 3 The temperature of the fluid $\left(T_{2}\right)$ in time when the MIP is exposed to increasing concentrations of L-nicotine $(0.05-10.0 \mu \mathrm{M}$ in PBS buffer, $\mathrm{pH}$ 7.4). The additions are indicated by arrows
In this formula, $\Delta T$ corresponds to the temperature difference $\left(T_{1}-T_{2}\right)$, and $P$ is the required heating power of the adjustable heat source in order to keep $T_{1}$ constant. With these parameters, the time-dependent $R_{\mathrm{th}}$ data can be calculated. These results are shown for the MIP functionalized electrode (Fig. 4a) and the electrode with only the adhesive MDMO-PPV (Fig. 4b). The NIP electrode was also measured, but not included in Fig. 4 since the results were very similar to the electrode with only the adhesive polymer.

Upon introducing a concentration of $10 \mu \mathrm{M}$ L-nicotine in PBS (pH 7.4), no response in $R_{\text {th }}$ is observed for the reference system. Surprisingly, the electrode functionalized with MIP particles showed a significant increase of $\sim 0.4{ }^{\circ} \mathrm{C} / \mathrm{W}$.

This can be explained qualitatively by the "poreblocking model." MIPs contain nanopores which can specifically rebind their target based on its size and functionality. Upon rebinding, the heat flux through one cavity is strongly reduced due to the presence of the template. As a result, the total heat transfer will be increased. The more L-nicotine will be bound, the more cavities will exhibit this behavior leading to an ultimately higher effect size. This "pore-blocking model" is schematically shown in Fig. 5.

In PBS, the $R_{\text {th }}$ stabilizes at $3.6 \pm 0.1{ }^{\circ} \mathrm{C} / \mathrm{W}$ and increases to $4.0 \pm 0.1{ }^{\circ} \mathrm{C} / \mathrm{W}$ upon addition of $10 \mu \mathrm{M}$ of $\mathrm{L}-$ nicotine (Fig. 4b). The effect size of $\sim 11.0 \%$ is significantly higher than the noise on the signal (3\%), thereby directly proving the binding of the target to the nanocavities of the MIP. These experiments were now performed with freshly prepared electrodes in the concentration regime $0-100 \mu \mathrm{M}$ L-nicotine in PBS. In order to demonstrate specificity of the sensor platform, the same measurements were also conducted with the NIPfunctionalized electrode. Additionally, the effect of cotinine (Fig. 1a) additions on the MIP was analyzed. This was done in order to address the selectivity, as cotinine is similar in chemical structure and L-nicotine's natural metabolite. The $R_{\text {th }}$ data can be represented as a dose-response curve, where the difference in $R_{\mathrm{th}}$ versus the concentration of the target is plotted. These results are summarized in Fig. 6.

The measurements were performed threefold; each measurement was conducted with a freshly functionalized electrode. The error bars given in Fig. 6 correspond to the standard deviation on the three separate experiments, showing excellent inter-reproducibility of the samples. We could measure in a wide concentration regime, from $0.2 \mu \mathrm{M}\left(\Delta R_{\mathrm{th}}=0.07 \pm 0.01{ }^{\circ} \mathrm{C} / \mathrm{W}\right)$ to $50 \mu \mathrm{M}\left(\Delta R_{\mathrm{th}}=0.60 \pm 0.03{ }^{\circ} \mathrm{C} / \mathrm{W}\right)$. The standard deviation at baseline level, when no L-nicotine is present $\left(0.01{ }^{\circ} \mathrm{C} / \mathrm{W}\right)$, is used to estimate the detection limit, 


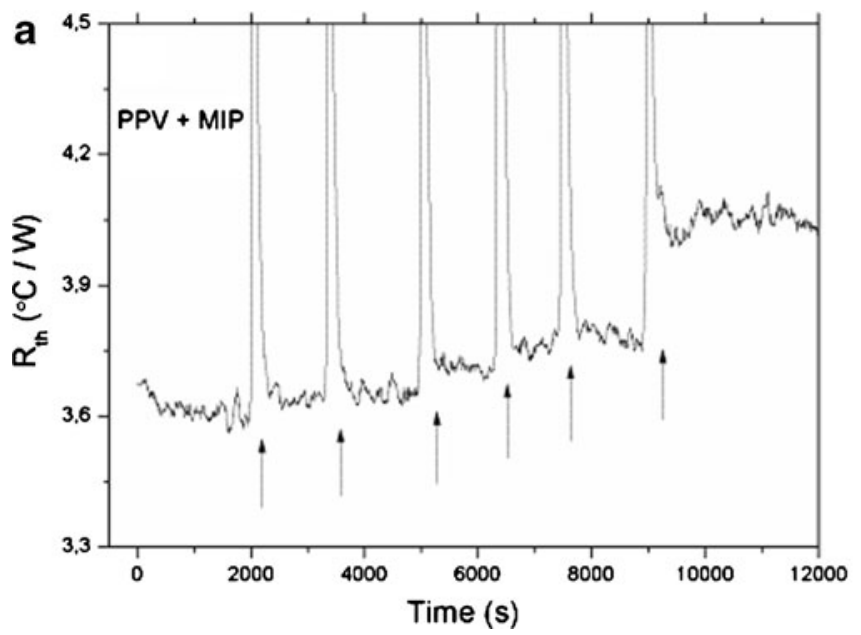

Fig. 4 Time dependence of the heat transfer resistance $R_{\mathrm{th}}$ upon exposure to increasing L-nicotine in PBS $(0.05,0.1,0.2,0.5,1.0$, and $10.0 \mu \mathrm{M}$ ) for a the MDMO-PPV-spincoated aluminum electrode functionalized with MIP particles by thermal treatment and $\mathbf{b}$ the aluminum

which is commonly defined as the concentration where the signal amplitude is three times the standard deviation. In the low concentration regime, $0.2-0.75 \mu \mathrm{M}$, the dose-response results can be represented well with a linear fit $\left(R^{2}=0.97\right)$. With this fit, the limit of detection was calculated to be approximately $125 \mathrm{nM}$. This is within the physiologically relevant range; the L-nicotine saliva concentration can vary from $0-500 \mu \mathrm{M}$ [36]. The sensing platform was also determined to be specific, since the NIP with L-nicotine and the MIP with cotinine did not show a significant response in $R_{\text {th }}$.

L-Nicotine in buffer: validation by impedance spectroscopy

After stabilizing in PBS, increasing concentrations of Lnicotine and cotinine were added $(0-1,000 \mu \mathrm{M})$. Between the addition steps, the sensor was left to stabilize for $10 \mathrm{~min}$. Subsequently, the response value was obtained by averaging
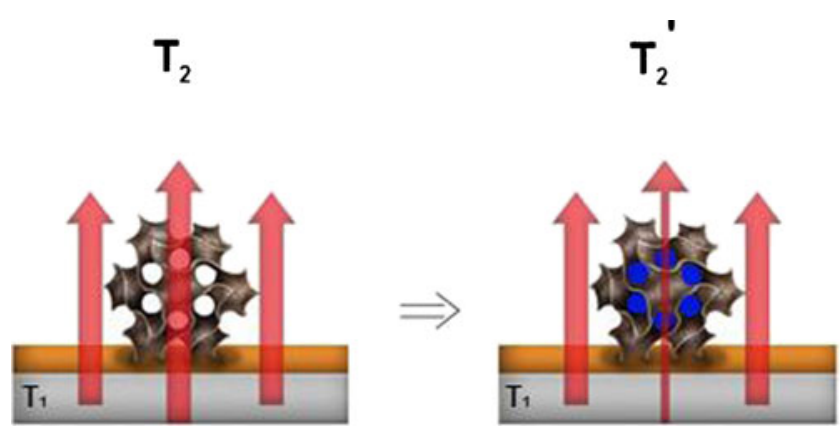

Fig. 5 Artist's impression of the "pore-blocking model." The MIP particle, embedded in the surface, contains various pores where binding sites are present for its template. When these channels are filled by target molecules (indicated by blue dots), heat flux through the MIP layer is strongly reduced

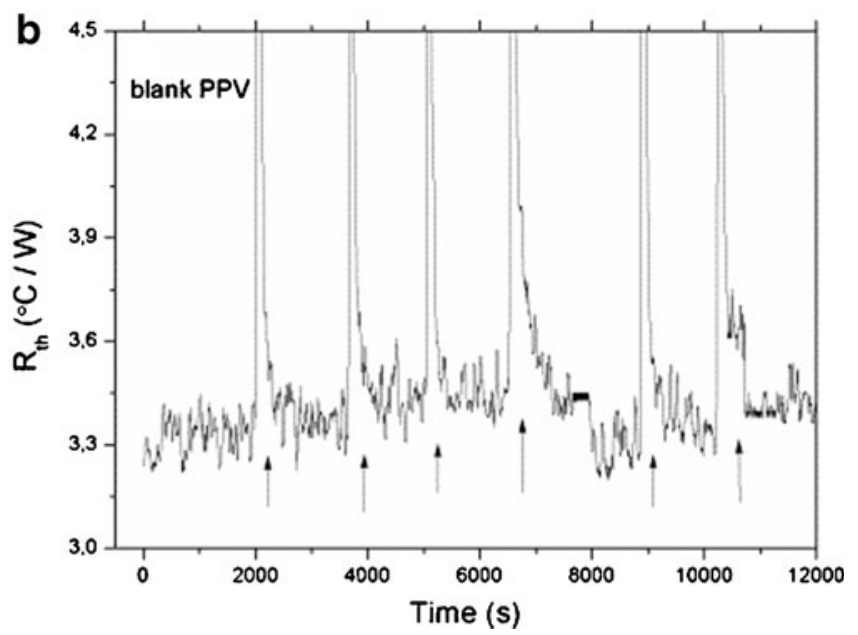

electrode spincoated with MDMO-PPV and subsequently heated above its glass transition temperature. To all the data, a percentile filter $(50 \%$ of 50 points) was applied

five impedance data points with an interval of $1 \mathrm{~min}$. All the obtained impedance data were normalized with respect to a starting value of $100 \%$ pure PBS. The corresponding doseresponse curves at a frequency $316 \mathrm{~Hz}$ are shown in Fig. 7 . This frequency was selected because it is low enough to probe capacitive effects and ensures a high signal-to-noise ratio [33]. The impedance was measured simultaneously with the heat transfer resistance, meaning Figs. 6 and 7 show the results of the same experiments which were obtained with a different readout technique.

Without the presence of L-nicotine, the standard deviation on the signal was $0.2 \%$. This would correspond to a

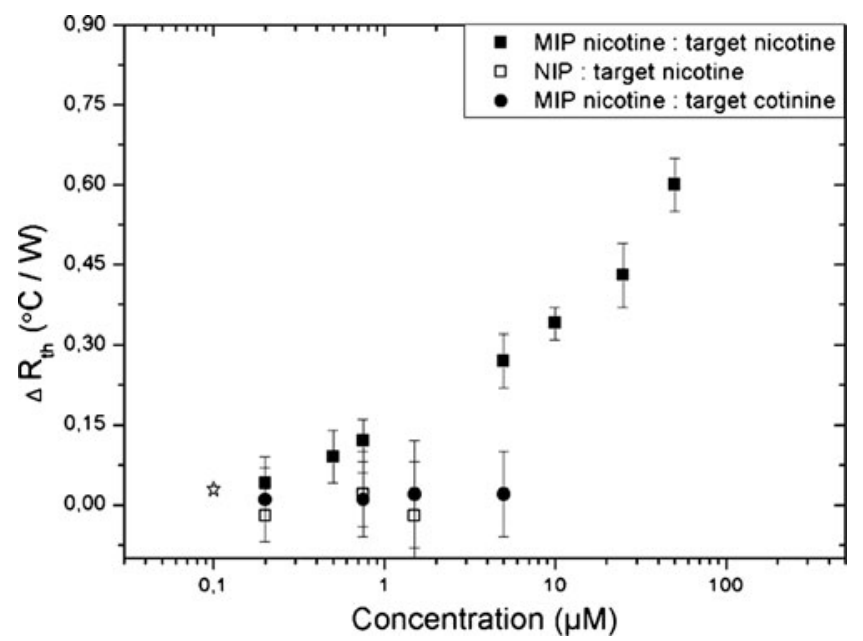

Fig. 6 Dose-response curve for the MIP (solid squares), NIP (open squares), and MIP with cotinine (solid circles), where $\Delta R_{\text {th }}$ is plotted versus the logarithm of the target concentration. The target concentration of L-nicotine and cotinine varies from 0 to $100 \mu \mathrm{M}$ in PBS (pH 7.4). The asterisk corresponds to the limit of detection; below this concentration, there is no significant response of the MIP to L-nicotine 


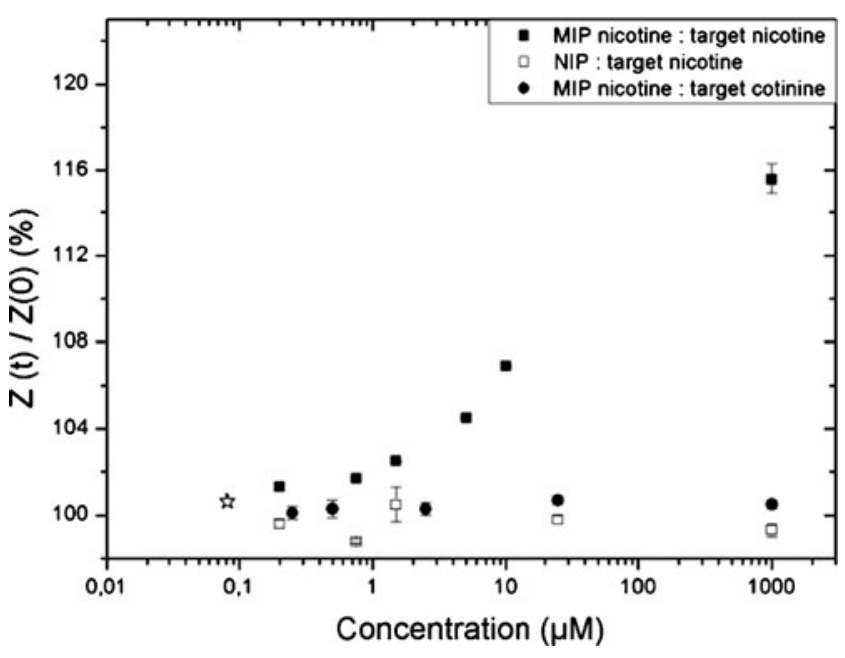

Fig. 7 Dose-response curve at $316 \mathrm{~Hz}$ for the MIP (solid squares), NIP (open squares), and MIP with cotinine (solid circles), where the normalized impedance is plotted versus the logarithm of the target concentration. These experiments were performed simultaneously with the heat transfer measurements, results of which are shown in Fig. 6. The concentration varies from 0 to $1,000 \mu \mathrm{M}$ of L-nicotine or cotinine in PBS (pH 7.4). The error bars are indicated, but might be smaller than symbol size

detection limit of approximately $100 \mathrm{nM}$, which is comparable to what was achieved with HTM. For the MIP, a linear response in $R_{\mathrm{th}}$ is observed from 0.1 to $2.5 \mu \mathrm{M} \mathrm{L}-$ nicotine. At higher concentrations, saturation is gradually occurring; the maximum response of $115.6 \pm 0.7 \%$ is obtained at $1,000 \mu \mathrm{M}$. For comparison, the NIP signal did not significantly change upon exposure to concentrations of L-nicotine while the increase of the MIP to $25 \mu \mathrm{M}$ cotinine was only $100.7 \pm 0.2 \%$. This corroborates with the heat transfer results, validating that the sensor

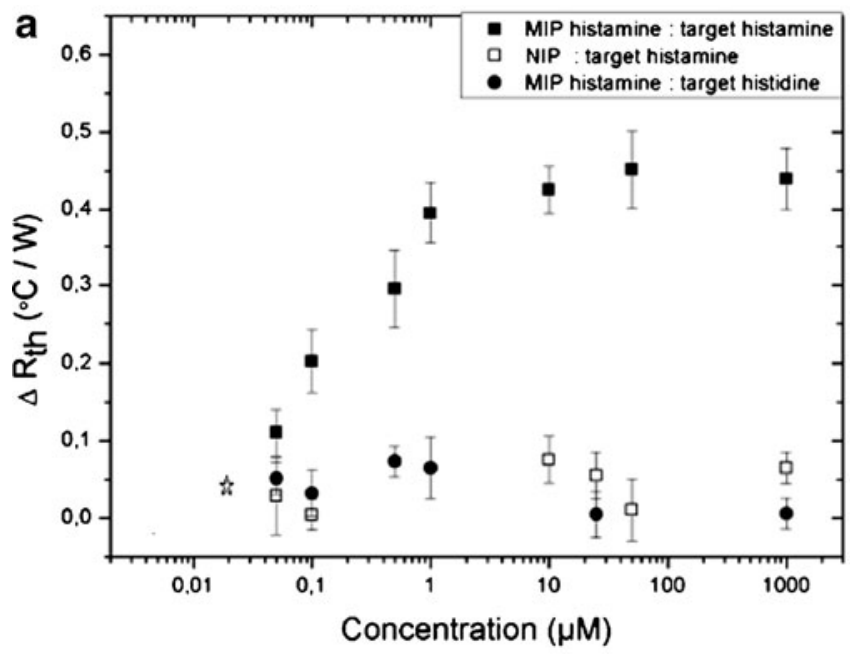

Fig. 8 a Dose-response curve for the MIP of histamine (solid squares), NIP (open squares), and MIP with histidine (solid circles), where $\Delta R_{\text {th }}$ is plotted versus the logarithmic target concentration. The target concentration varies from 0.2 to $1,000 \mu \mathrm{M}$ in PBS ( $\mathrm{pH}$ 7.4). b platform can be applied for the specific detection of Lnicotine in buffer solutions.

Histamine and serotonin measurements in buffer

To demonstrate the applicability of the sensor platform for a variety of target molecules, additional histamine and serotonin measurements $(0-1,000 \mu \mathrm{M}$ in PBS of $\mathrm{pH}$ 7.4) were performed with MIP materials presented in previous work $[10,20]$. As analogs, we selected for histamine its precursor histidine (Fig. 1b) and, for serotonin, its natural competitor dopamine (Fig. 1c). The dose-response curves are shown in Fig. 8.

Figure 8a shows the dose-response curve for histamine. At a maximum concentration of $1,000 \mu \mathrm{M}$ histamine, the $\Delta R_{\mathrm{th}}$ of the MIP increased with $0.4 \pm 0.04{ }^{\circ} \mathrm{C} / \mathrm{W}$. Its references, the NIP with histamine and the histamine MIP with histidine as target, showed no significant response at this concentration range. Thereby, it is proven that the sensing platform can detect histamine in a specific manner. Furthermore, we estimated the detection limit to be approximately $30 \mathrm{nM}$, which is within the range as was obtained previously with impedance spectroscopy [21]

From Fig. $8 \mathrm{~b}$ can be determined that upon addition of $50 \mu \mathrm{M}$ serotonin, the $R_{\mathrm{th}}$ of the MIP goes up by $1.07 \pm 0.03{ }^{\circ} \mathrm{C} / \mathrm{W}$. There was no effect on the NIP; some minor increases occurred for the serotonin MIP in combination with the competitor dopamine $\left(0.2 \pm 0.05{ }^{\circ} \mathrm{C} / \mathrm{W}\right)$. However, the signal ratio between target versus competitor is approximately 5 , which ensures selective detection of serotonin with the sensor platform. The estimated limit of detection is $20 \mathrm{nM}$, low enough to measure in the physiologically relevant concentration range [20]

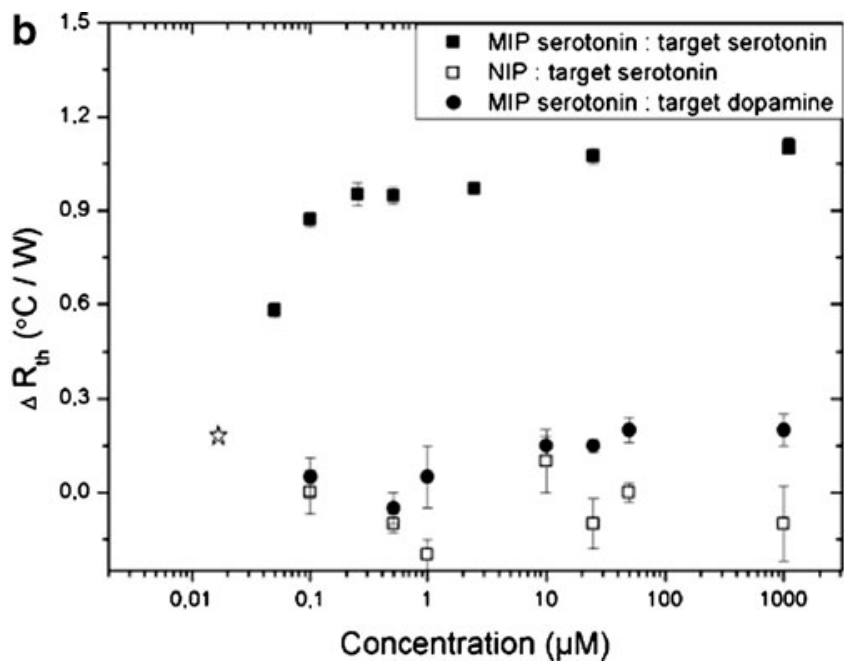

Dose-response curve for the MIP of serotonin (solid squares), NIP (open squares), and MIP with dopamine (solid circles), where $\Delta R_{\text {th }}$ is plotted versus the logarithmic target concentration. The concentrations in PBS vary from 0.2 to $1,000 \mu \mathrm{M}$ 
Table 1 LOD in $1 \times$ PBS with HTM and impedimetric readout compared to the biologically relevant concentration

\begin{tabular}{|c|c|c|c|c|c|}
\hline \multirow{3}{*}{ Target } & \multirow{2}{*}{\multicolumn{2}{|c|}{$\frac{1 \times \mathrm{PBS}}{\mathrm{LOD}}$}} & \multicolumn{3}{|l|}{ Biological samples } \\
\hline & & & \multirow[t]{2}{*}{ Saliva } & \multirow[t]{2}{*}{ Urine } & \multirow[t]{2}{*}{ Blood } \\
\hline & HTM & Impedance & & & \\
\hline L-Nicotine & $100 \mathrm{nM}$ & $100 \mathrm{nM}[34]$ & $0.2-1,000 \mu \mathrm{M}[36]$ & $0.3-10 \mu \mathrm{M}[36]$ & - \\
\hline Serotonin & $20 \mathrm{nM}$ & $5 \mathrm{nM}[20]$ & - & - & $10-1,500 \mathrm{nM}[20]$ \\
\hline Histamine & $30 \mathrm{nM}$ & $15 \mathrm{nM}[21]$ & - & $200-750 \mathrm{nM}[10]$ & $10-1,000 \mathrm{nM}[10]$ \\
\hline
\end{tabular}

The detection limits of the HTM and impedimetric readout for various targets are summarized in Table 1. Furthermore, the biologically relevant concentration range is given.

In order to assess the applicability of the sensor platform in biological media, saliva samples spiked with L-nicotine $(0.25,0.5,1.0,2.5$, and $10.0 \mathrm{mM})$ were evaluated. For these measurements, there was no need for dilution, thereby further simplifying the sample preparation. The absolute $R_{\mathrm{th}}$ values for the MIP and NIP after exposure to increasing spiked concentrations of L-nicotine are shown in Fig. 9.

The absolute $R_{\mathrm{th}}$ values in saliva are higher than for the Lnicotine MIP in PBS. This can be due to two reasons; first, the absorbed proteins act as an additional insulating layer, increasing the total thermal resistance, and second, the viscosity of the saliva is much higher which limits the heat transport and prolongs measurement time.

Figure 9 shows that there was no significant response of the NIP to increasing concentrations of spiked L-nicotine. For the MIP, in the regime from 0.25 to $1.0 \mathrm{mM}$, the increase in $\Delta R_{\mathrm{th}}$ is linear $\left(R^{2}=0.97\right)$ with a maximum of $0.5 \pm 0.01{ }^{\circ} \mathrm{C} / \mathrm{W}$. For concentrations higher than $1.0 \mathrm{mM}$, saturation is occurring due to increasing occupation of the

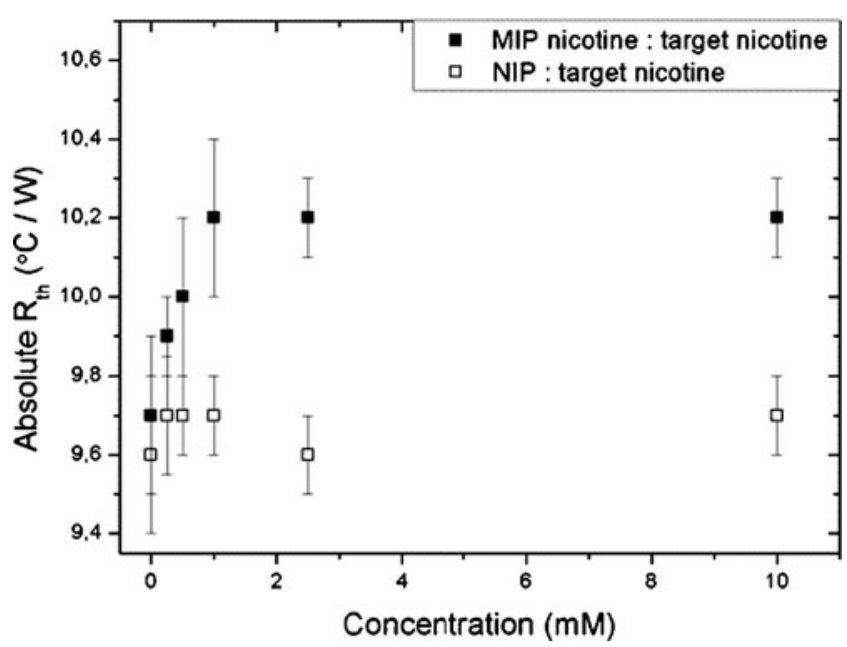

Fig. 9 The dose-response curve for the concentration of L-nicotine in spiked saliva $(0,0.25,0.5,1,2,5,10 \mathrm{mM})$ versus the differential heat transfer resistance $\left(\Delta R_{\mathrm{th}}\right)$. This curve represents the absolute values of the $R_{\mathrm{th}}$ of MIP and NIP binding sites. The sensitive regime of the sensor is between 0.25 and $1.0 \mathrm{mM}$, and these concentrations are within the relevant range of biological samples [36]. Therefore, the applicability of the sensor platform for measurements in biological samples is demonstrated.

In the case of saliva, no reference impedance tests could be conducted. This is due to the absorption of proteins, resulting in an extremely high electrical resistance beyond the measuring limit. While we could not directly validate the results, this might be an additional benefit for the $R_{\mathrm{th}}$-based sensor platform as we can also measure in complicated viscous solutions.

\section{Conclusions}

In this article, we presented the HTM for the specific detection of L-nicotine. This is the first time detection of small molecules based on MIP-type receptors in combination with the HTM concept has been reported. The principle of the technique can be explained by the "pore-blocking model"; upon binding of the target to the nanocavities present in the MIP, heat transport in that direction is strongly reduced resulting in a total increase of the heat transfer resistance. For proof-of-principle measurements, L-nicotine concentrations in PBS solutions were analyzed. The detection could be performed in a specific manner, which was validated by reference tests with impedimetric readout. In addition, a similar detection limit was achieved compared to the impedance spectroscopy tests. This detection limit is surprising low; it is in the nanomolar range which is well within the physiologically relevant regime. Furthermore, it was possible to extend this method to other small molecules, which was proven for histamine and serotonin samples in buffer solutions. As a first proof-of-application experiment, saliva samples spiked with L-nicotine were evaluated. The constructed dose-response curve showed sensitivity in the physiologically relevant regime, demonstrating the applicability of the sensor platform in biological media. Summarizing, the novel approach HTM enables the fast, straightforward, and low-cost detection of small molecules with MIP receptors which makes it of great interest for biosensing and analytical applications. 
Acknowledgments This work is supported by the Life-Science Initiative of the Province of Limburg (M. Peeters) and by the Internationalization Program of Universidade de São Paulo, Brazil (P. Csipai). The authors also would like to thank A. Gaulke and P. Losada-Pérez for stimulating scientific discussions and H. Penxten, J. Soogen, C. Willems, and J. Baccus for technical assistance.

\section{References}

1. Mosbach K (1994) Molecular imprinting. Trends Biochem Sci 19:9-14

2. Arshady R, Mosbach K (1981) Synthesis of substrate-selective polymer by host-guest polymerization. Chem Phys 182:687-692

3. Shi H, Tsai WB, Garrison MD, Ferrari S, Ratner BD (1999) Template-imprinted nanostructured surfaces for protein recognition. Nature 398:593-597

4. Hayden O, Lieberzeit PA, Blaas D, Dickert FL (2006) Artificial antibodies for bioanalyte detection-sensing viruses and proteins. Adv Funct Mater 16:1269-1278

5. Hayden O, Mann KJ, Krassnig S, Dickert FL (2006) Biomimetic ABO blood-group typing. Angew Chem Int Ed 45:2626-2629

6. Jenik M, Seifner A, Lieberzeit PA, Dickert FL (2009) Pollenimprinted polyurethanes for QCM allergen sensors. Anal Bioanal Chem 394:523-528

7. Wulff G (1993) The role of binding site interactions in the molecular imprinting of polymers. Trends Biotechnol 11:85-87

8. Vlatakis G, Andersson LI, Müller R, Mosbach K (1993) Drug assay using antibody mimics made by molecular imprinting. Nature 361:645-647

9. Owens K, Karlsson L, Lutz ESM, Andersson LI (1999) Molecular imprinting for bio- and pharmaceutical analysis. TrAC, Trends Anal Chem 18:146-154

10. Horemans F, Alenus J, Bongaers E, Weustenraed A, Thoelen R, Duchateau J, Lutsen L, Vanderzande D (2010) MIP-based sensor platforms for the detection of histamine in the nano- and micromolar range in aqueous media. Sens Actuators B 148:392-398

11. Vaihinger D, Landfester K, Kräuter I, Brunner H, Tovar G (2002) Molecularly imprinted polymer nanospheres as synthetic receptors obtained by miniemulsion polymerization. Macromol Chem Phys 203:1965-1973

12. Stobiecka M, Deeb J, Hepel M (2009) Molecularly templated polymer matrix films for biorecognition processes: sensors for evaluating oxidative stress and redox buffering capacity. ECS Trans 19:15-32

13. Haupt K, Mosbach K (2000) Molecularly imprinted polymers and their use in biomimetic sensors. Chem Rev 100:2495-2504

14. Benito-Pena E, Urraca JL, Sellergren B, Moreno-Bondi MC (2008) Solid-phase extraction of fluoroquinolones from aqueous samples using a watercompatible stochiometrically imprinted polymer. J Chromatogr A 1208:62-70

15. Andersson LI, Miyabayashi A, O'Shannessy DJ, Mosbach K (1990) Enantiomeric resolution of amino acid derivatives on molecularly imprinted polymers as monitored by potentiometric measurements. J Chromatogr 516:323-331

16. Vidyasankar S, Ru M, Arnold FH (1997) Chiral ligand exchange adsorbents for amines and underivatized amino acids: 'bait-andswitch' molecular imprinting. J Chromatogr A 775:51-63

17. Avila M, Zougagh M, Escarpa A, Rios A (2008) Molecularly imprinted polymers for selective piezoelectric sensing of small molecules. Trends Anal Chem 27:54-65

18. Piletsky SA, Turner APF (2002) Electrochemical sensors based on molecularly imprinted polymers. Electroanalysis 13:317-323
19. Mao Y, Bao Y, Gan S, Li F, Niu L (2011) Electrochemical sensor for dopamine based on a novel graphene-molecular imprinted polymers composite recognition element. Bios Bioelectron 28:291-297

20. Peeters M, Troost FJ, van Grinsven B, Horemans F, Alenus J, Murib MS, Keszthelyi D, Ethirajan A, Thoelen R, Cleij TJ, Wagner P (2012) MIP-based biomimetic sensor for the electronic detection of serotonin in human blood plasma. Sens Actuators B 171-172:602-610

21. Peeters M, Troost FJ, Mingels RHG, Welsch T, van Grinsven B, Vranken T, Ingebrandt S, Thoelen R, Cleij TJ, Wagner P (2013) Impedimetric detection of histamine in bowel fluids using synthetic receptors with $\mathrm{pH}$-optimized binding characteristics. Anal Chem 85:1475-1483

22. Patel AK, Sharma PS, Prasad BB (2010) Trace-level sensing of creatine in real sample using a zwitterionic molecularly imprinted polymer brush grafted to sol-gel modified graphite electrode. Thin Solid Films 10:2847-2853

23. Prasad BB, Srivastava S, Tiwari K, Sharma PS (2009) Trace-level sensing of dopamine in real samples using molecularly imprinted polymer-sensor. Biochem Eng J 44:232-239

24. Hoshino Y, Koide H, Furuya K, Haberaecker WW III, Lee SH, Kodama T, Kanazawa H, Oku N, Shea KJ (2010) Recognition, neutralization, and clearance of target peptides in the bloodstream of living mice by molecularly imprinted polymer nanoparticles: a plastic antibody. J Am Chem Soc 132:6644-6645

25. van Grinsven B, Vanden Bon N, Strauven H, Grieten L, Murib MS, Jiménez Monroy KL, Janssens SD, Haenen K, Schöning M, Vermeeren V, Ameloot M, Michiels L, Thoelen R, De Ceuninck W, Wagner P (2012) Heat-transfer resistance at solid-liquid interfaces: a tool for the detection of single-nucleotide polymorphisms in DNA. ACS Nano 6:2712-2721

26. Athikomrattanakul U, Gajovic-Eichelmann N, Scheller FW (2011) Thermometric sensing of nitrofurantoin by noncovalently imprinted polymers containing two complementary functional monomers. Anal Chem 83:7704-7711

27. Lettau K, Katterle M, Warsinke A, Scheller FW (2006) A bifunctional molecularly imprinted polymer (MIP): analysis of binding and catalysis by a thermistor. Angew Chem Int Edit 45:6986-6990

28. Rajkumar R, Katterle M, Warsinke A, Möhwald H, Scheller FW (2008) Thermometric MIP sensor for fructosyl valine. Biosens Bioelectron 23:1195-1199

29. Neal LB (1997) The role of nicotine in smoking-related cardiovascular disease. Prev Med 26:412-417

30. Schrek R, Baker LA, Ballard GP, Dolgoff S (1950) Tobacco smoking as an etiologic factor in disease. I Cancer Cancer Res 10:49-58

31. Doll R, Peto R (1981) The causes of cancer: quantitative estimates of avoidable risks of cancer in the United States today. JNCI 66:1191-1308

32. Mayer AS, Newman LS (2001) Genetic and environmental modulation of chronic obstructive pulmonary disease. Respir Physiol 128:3-11

33. Bergström J (2004) Tobacco smoking and chronic destructive periodontal disease. Odontology 92:1-8

34. Thoelen R, Vansweevelt R, Duchateau J, Horemans F, D'Haen J, Lutsen L, Vanderzande D, Ameloot M, van de Ven M, Cleij TJ, Wagner P (2008) A MIP-based impedimetric sensor for the detection of low-MW molecules. Biosens Bioelectron 23:913-918

35. Louwet D, Vanderzande J, Gelan A (1995) A general synthetic route to high-molecular-weight poly(p-xylene)-derivatives - a new route to poly (p-phenylene vinylene. Synth Met 69:509-510

36. Russell MA, Wilson C, Feyerabend C, Cole PV, Salojee Y (1976) Nicotine chewing gum as a substitute for smoking. Br Med J 6017:1043-1046 